\title{
Research Note \\ The rotation period of 804 Hispania: Some considerations on its nature
}

\author{
M. Calabresi and G. Roselli
}

Associazione Romana Astrofili, Frasso Sabino Observatory, Casella postale 4011, 00100 Rome, Italy

Received 11 February 2000 / Accepted 28 November 2000

\begin{abstract}
Photometric observations of 804 Hispania performed at the Teide Observatory during the opposition in September 1998 are presented and analyzed. A rotation period of $7.405 \pm 0.010 \mathrm{~h}$ was derived. It was possible to confirm that the lightcurve presents two maxima and minima. We discuss in detail some considerations on its nature.
\end{abstract}

Key words. micro planets, asteroids - solar system: general

\section{Introduction}

The asteroid 804 Hispania was observed during its opposition in September 1998 when its magnitude was $V=10.8$. This is a $\mathrm{C}$ type asteroid, which is a very common type with a $161 \mathrm{Km}$ diameter (Tedesco 1989) Photoelectric measurements to obtain the light curve and rotation period have been reported by several authors (Harris 1983; Debehogne 1983; Magnusson et al. 1991); however, the period value has not been unambiguously determined; at least two values have been proposed (14.851 $\mathrm{h}$ and $7.42 \mathrm{~h}$ ) We aimed to determine the period and to confirm the presence of a secondary minimum in the light curve.

\section{Observations}

Observations were performed during 5 nights at the Observatorio del Teide (Canary Islands-Spain) using the MONS telescope, a $0.5 \mathrm{~m}$ diameter Cassegrain equipped with a photoelectric photometer. The photomultiplier tube was a Hamamatsu 1P21-P, a very low dark current device (final test sheet from Hamamatsu gives a $0.07 \mathrm{nA}$ dark current at a temperature of $25^{\circ} \mathrm{C}$ ). $V$ and $B$ band data were reduced in the standard way. Expected data for the observed asteroid are given in Table 1, where the heliocentric and geocentric distance $r$ and $\Delta$ are measured in astronomical units and $\alpha$ is the Solar phase angle. Comparison stars' data are reported in Table 2 (from the Tycho catalogue).

Send offprint requests to: M. Calabresi, e-mail: MC7851@mclink.it
Table 1. 804 Hispania expected data

\begin{tabular}{|c|c|c|c|c|c|}
\hline DATE $0^{\mathrm{h}} \mathrm{UT}$ & $\mathrm{RA}_{2000}$ & $\mathrm{DEC}_{2000}$ & $\Delta$ & $r$ & $\alpha$ \\
\hline 1998 Sep. 18 & 23:08:12.377 & $-04: 36: 09.57$ & 1.454 & 2.452 & 3.53 \\
\hline 1998 Sep. 19 & 23:07:12.412 & $-04: 34: 35.55$ & 1.457 & 2.453 & 4.02 \\
\hline 1998 Sep. 20 & 23:06:13.108 & $-04: 32: 50.68$ & 1.460 & 2.453 & 4.50 \\
\hline 1998 Sep. 21 & 23:05:14.538 & $-04: 31: 21.75$ & 1.463 & 2.454 & 4.98 \\
\hline 1998 Sep. 22 & 23:04:16.770 & $-04: 29: 41.60$ & 1.467 & 2.454 & 5.46 \\
\hline 1998 Sep. 23 & 23:03:19.873 & $-04: 27: 59.03$ & 1.471 & 2.455 & 5.93 \\
\hline
\end{tabular}

Table 2. Comparison stars' data

\begin{tabular}{cccccc}
\hline Object & $\mathrm{RA}_{2000}$ & Dec $_{2000}$ & $V$ & $B-V$ & Type \\
\hline SAO 146467 & $23: 02: 37.641$ & $-03: 59: 24.98$ & 9.83 & 1.472 & K2 \\
SAO 146448 & $23: 01: 23.211$ & $-04: 22: 21.93$ & 9.57 & 0.378 & F8 \\
\hline
\end{tabular}

\section{Analysis}

The light curve in the $V$ band was obtained after reducing the data in the classical way: the apparent magnitude was first corrected for distance and related to $H$, the magnitude at zero phase angle by:

$H(\alpha)=H-2.5 \log \left((1-G) \Phi_{1}(\alpha)+G \Phi_{2}(\alpha)\right)$,

here $G$ is the slope parameter which gives an indication of the gradient of the phase curve and $\Phi_{1}, \Phi_{2}$ are two specified phase functions derived empirically (Bowell et al. 1989). In our case we assumed $G=0.09 \pm 0.02$ (given by Harris \& Young 1983); the accuracy of measurement is \pm 0.02 magnitude in $V$ band. 
Table 3. Frequencies identification

\begin{tabular}{ccccc}
\hline & Frequency & Identification & $\Theta_{\mathrm{ob}}$ & $p$ value \\
\hline $\mathrm{a}$ & 3.241 & $f_{0}$ & 0.25 & 0.00 \\
$\mathrm{~b}$ & 2.184 & $2 / 3 f_{0}$ & 0.35 & 0.00 \\
$\mathrm{c}$ & 1.625 & $f_{0} / 2$ & 0.51 & 0.00 \\
\hline
\end{tabular}

\section{Results}

The period was detected using the Phase Dispersion Minimization technique (Stellingwerf 1978), which is particularly suitable in cases where only a few observations are available during a short time and with a non-sinusoidal light curve. This method tries to minimize the $\Theta$ statistic, which is a measure of the dispersion of observed data round a mean light curve related to a trial frequency. We used the Algol software package, developed by members of GEOS (P. Bernasconi and A Gaspani). The main frequency was first determined by a rapid scan and subsequently verified with further testing of accuracy.

The PDM technique finds all the periodic components; the master frequency normally corresponds to a minimum value of $\Theta$ at the regions in the periodogram where the subharmonics also appear $\left(f_{n}=f_{0} / n\right.$, where $f_{0}$ is the principal frequency and $n$ is an integer $>1$ ); aliased frequencies appear as harmonics of frequency $f \pm 1, f \pm 1 / 2$, $f \pm 1 / 3, \ldots, 2 f \pm 1,2 f \pm 1 / 2$.

To determine the statistical significance of the periods derived using phase -dispersion -minimization, we apply the randomization test proposed by Nemec \& Nemec (1985) to verify the null hypothesis that the period is equal to a determinate value. The software is in the Matlab package and was adapted by the authors using some scripts by Dr. Eran O. Ofek.

The $\Theta_{\mathrm{ob}}\left(P_{0}\right)$, corresponding to the trial period $P_{0}$ evaluated for the observed data, was compared to the $\Theta\left(P_{0}\right)$ of random ordered data, with the hypothesis that if there is no periodicity in the observed data, the magnitude is independent of observation time.

The $p$ value is the proportion of permutations that give a value of $\Theta\left(P_{0}\right)$ less than or equal to $\Theta_{\mathrm{ob}}\left(P_{0}\right)$. If the null hypothesis is true, the difference between $\Theta\left(P_{0}\right)$ and $\Theta_{\mathrm{ob}}\left(P_{0}\right)$ is negligible and $p$ is not small; on the other hand, if a periodic structure is present, the difference between $\Theta\left(P_{0}\right)$ and $\Theta_{\mathrm{ob}}\left(P_{0}\right)$ is relevant and $p$ is negligible.

In our test we used $m=5000$ random permutation; in Table 3 we identify the frequency corresponding to a relevant peak of $\Theta_{\mathrm{ob}}$. As a final test to eliminate spurious value, we searched for frequencies with a $p$ value $<0.01$ (95\% confidence interval 0.0 to 0.01 using a binomial distribution ). Only the main frequency gave us a rotational phase curve; using other frequencies we obtained a random distribution of the observed data.

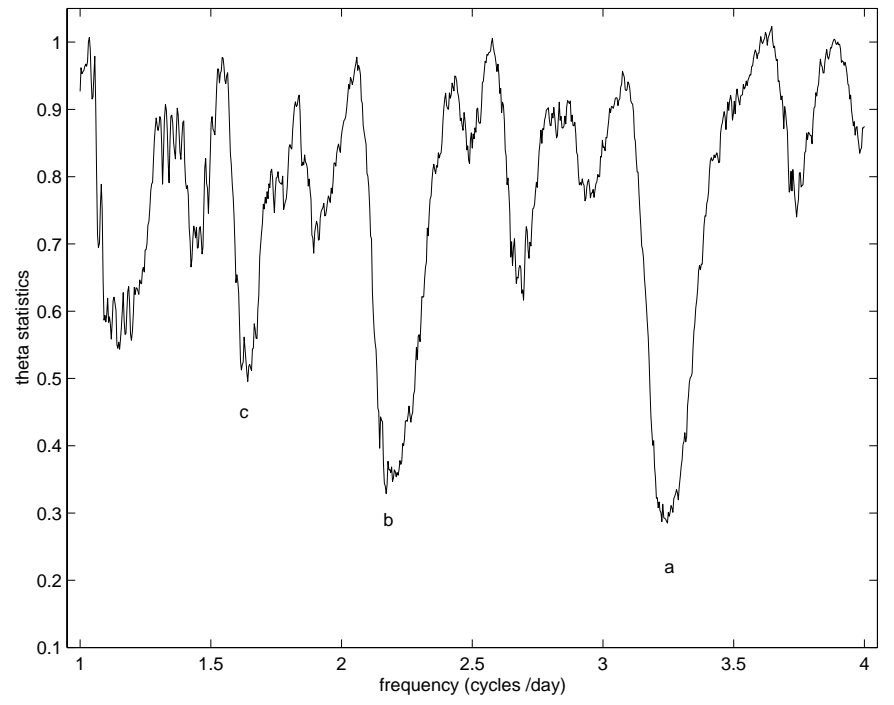

Fig. 1. Periodogram

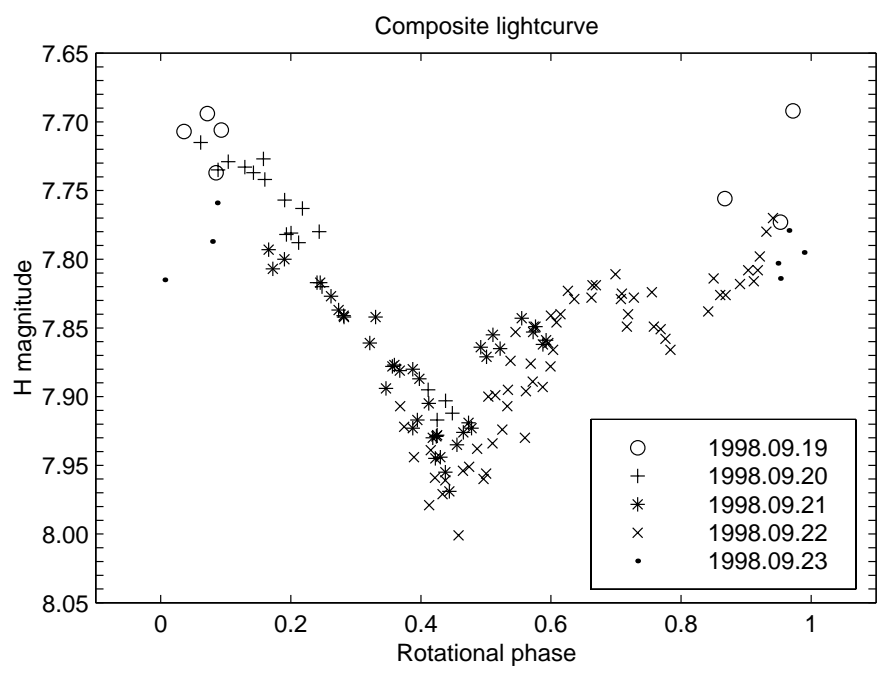

Fig. 2. Composite lightcurve with zero phase at UT 1998 September 20

The main frequency value was estimated at $3.241 \pm$ 0.005 cycles per day corresponding to a synodic period of $7.405 \pm 0.010 \mathrm{~h}$, in accordance with Debehogne et al. (1983) who found $7.42 \mathrm{~h}$. This value is also close to the half period found by Harris \& Young (1983).

In Fig. 1 we show the $\Theta$ transform versus frequency in cycles per day $\left(f_{\min }=1, f_{\max }=4\right.$; resolution $\Delta f=$ $0.0035)$.

In Fig. 2 we report the composite lightcurve corresponding to a $7.405 \pm 0.010 \mathrm{~h}$ period and confirm that the lightcurve shows two maxima and two minima.

Debehogne et al. (1983) discuss this light curve and report two distinct hypotheses: a particular parallelepiped like asteroid shape or a binary nature.

Applying the procedure proposed by Leone et al. (1984) it is possible to obtain a binary model from the observational parameter spin period and light curve amplitude. 
We performed the analysis using the network of Roche sequences in the plane $A-\omega$, the rotational parameter $T=7.405\left(T=\frac{2 \pi}{\omega}\right.$ in hours $)$ and $A=0.2(A=$ lightcurve amplitude of the system). Our results show that the model used may be consistent with a binary system whose mass ratio between the two components is $q=0.1$ and a density $3 \leq \rho \leq 5 \mathrm{~g} \mathrm{~cm}^{-3}$ ( $\rho$ density assumed to be equal for both components).

A density of $3 \mathrm{~g} \mathrm{~cm}^{-3}$ is the upper limit for a $\mathrm{C}$ type asteroid so we could not rule out the binary nature of 804 even if the probability is low.

\section{Conclusions}

We detected synodic period of $7.405 \mathrm{~h}$ for the asteroid 804 Hispania. In addition, we found the value of $H=7.90$ to be in agreement with the IRAS observation $H=7.87$ and a $B-V=0.73 \pm 0.05$. We note that there is some possibility that the asteroid may be a double body with a mass ratio of 0.1 . We are aware that a definitive solution to this problem will only be found by different observation techniques, such as occultations or speckle interferometry.

Acknowledgements. The authors would like to thank the Director of Instituto de Astrofisica de Canarias Prof. Francisco Sànchez for the use of the Mons telescope and the technical staff of Teide Observatory for the assistance during the observations. The Mons telescope is operated by the Instituto de
Astrofisica de Canarias (IAC) at the Spanish Observatorio del Teide (Tenerife) of the IAC.

The authors are also very grateful to Miss Monica Murphy for the logistical assistance in preparing the observation expedition.

Finally, we thank Dr. A. W. Harris and Dr. J. L. Hilton for the suggestions on the paper and for helpful discussions.

\section{References}

Bowell, E., Gehrels, T., \& Zellner, B. 1979, in Asteroids, ed. T. Gehrels (Univ. of Arizona), 1108

Cellino, A., Pannunzio, R., Zappalà, V., Farinella, P., \& Paolicchi, P. 1985, A\&A, 144, 355

Chandrasekhar, S. 1969, Ellipsoidal Figures of Equilibrium (Yale Univ. Press, New Haven, London)

Debehogne, H., De Sanctis, G., \& Zappala, V. 1983, Icarus, 55, 236

Devies, S. R. 1990, MNRAS, 244, 93

Gaspani, A. 1998, Anspec, GEOS

Harris, \& Young 1983, Icarus, 54, 59

Leone, G., Farinella, P., Paolicchi, P., \& Zappalà, V. 1984, A\&A, 140, 265

Magnusson, P., Lagerkvist, C.-I. 1991, A\&AS, 87, 269

Nemec, A. F. L., \& Nemec, J. M. 1985, AJ, 90, 2317

Stellingwerf, R. F. 1978, ApJ, 224, 953

Tedesco, E. F. 1989, in Asteroids II, ed. R. P. Binzel, T. Gehrels, \& M. Matthews (Univ. of Arizona), 1104

Zappalà, V. 1980, Moon and Planets, 23, 345

Zappalà, V., Scaltriti, F., Farinella, P., \& Paolicchi, P. 1980, Moon and Planets, 22, 153 